\title{
Dependence of spin torque switching probability on electric current
}

\author{
Tomohiro Taniguchi ${ }^{1,2}$ and Hiroshi Imamura* \\ ${ }^{1}$ Magnetic Materials Unit, National Institute for Materials Science, 1-2-1 Sengen, Tsukuba 305-0047, Japan \\ ${ }^{2}$ Nanoscale Theory Group, Nanosystem Research Institute, \\ National Institute of Advanced Industrial Science and Technology, 1-1-1 Umezono, Tsukuba 305-8568, Japan
}

(Dated: June 24, 2018)

\begin{abstract}
Dependence of the thermally assisted spin torque switching probability on the sweep electric current was investigated theoretically. The analytical expressions of the switching times for $b=1$ and $b=2$ are derived based on the rate equation, where $b$ is the exponent of the current term in the switching rate. The switching current is approximately proportional to the temperature $T$ and the logarithm of the sweep rate $v$ for both $b=1$ and $b=2$ in the experimentally performed ranges of $T$ and $v$. Experiments in very low temperature range are required to determine the exponent $b$.
\end{abstract}

\section{INTRODUCTION}

Spin random access memory (Spin RAM), which employs spin torque switching [1, 2] as the writing method, is one of the key spin-electronics applications for future nanotechnology. In Spin RAM application, a high thermal stability $\Delta_{0}$ of more than 40 is required to guarantee retention time longer than 10 years. Recently, Hayakawa et al. and Yakata et al. studied the thermal stabilities of antiferromagnetically and ferromagnetically coupled synthetic free layers respectively, and found higher thermal stabilities compared with that of a single free layer [3 5].

The thermal stability is determined by the thermally assisted magnetization switching probability under the effect of the applied magnetic field or spin torque. The magnetic field or spin torque is assumed to be small compared to the anisotropy field $H_{\mathrm{K}}$ or critical current $I_{\mathrm{c}}$ at $T=0$. In 1963 Brown derived the analytical expression of the switching probability which includes the effect of constant field $H_{\text {appl }}$ [6] which is given by $P=1-\exp \left\{-f_{0} t \exp \left[-\Delta_{0}\left(1-H_{\text {appl }} / H_{\mathrm{K}}\right)^{2}\right]\right\}$, where $f_{0}$ is the attempt frequency. The switching probability under the effect of constant current $I$ (spin torque) was independently derived by Koch et al. 7] and Li and Zhang [8] in 2004, and is given by $P=1-\exp \left\{-f_{0} t \exp \left[-\Delta_{0}(1-\right.\right.$ $\left.\left.\left.H_{\text {appl }} / H_{\mathrm{K}}\right)^{2}\left(1-I / I_{\mathrm{c}}\right)\right]\right\}$. It should be noted that the exponent of the current term, $\left(1-I / I_{\mathrm{c}}\right)$, in this formula is unity. On the other hand, Suzuki et al. showed that the switching probability is given by $P=1-$ $\exp \left\{-f_{0} t \exp \left[-\Delta_{0}\left(1-H_{\mathrm{appl}} / H_{\mathrm{K}}-I / I_{\mathrm{c}}\right)^{2}\right]\right\}[9]$, where the exponent of the current term is 2 . Recently, we derived the switching probability formula which is given by $P=1-\exp \left\{-f_{0} t \exp \left[-\Delta_{0}\left(1-H_{\text {appl }} / H_{\mathrm{K}}\right)^{2}\left(1-I / I_{\mathrm{c}}\right)^{2}\right]\right\}$ [10], which slightly different compared to the result of Suzuki et al. but agrees well with the results of Butler et al. [11]. The determination of the value of the exponent $b$ of the current term in the switching probability formula, $P=1-\exp \left\{-f_{0} t \exp \left[-\Delta_{0}\left(1-H_{\text {appl }} / H_{\mathrm{K}}\right)^{2}\left(1-I / I_{\mathrm{c}}\right)^{b}\right]\right\}$, remains unsettled, although it is very important for the determination of the thermal stability [10].

*Electronic address: h-imamura@aist.go.jp
Recently we [10] pointed out the reason why the values of the exponent $b$ for the spin torque switching are different between Ref. [7] and Ref. [10]. In the case of the completely in-plane switching of the in-plane magnetized system, or in the case of the perpendicularly magnetized system, the switching can be described by only the angle from the easy axis, and the effect of spin torque can be regarded as the enhancement of the applied field, as shown in Ref. [9-11]. Thus, the exponent $b$ is reduced to 2 , as is in the case for the field switching [6]. However, the results of Refs. 7, [8] $(b=1)$ are supported by the experimental result 12], in which the mean switching current is approximately proportional to the temperature $T$ and the logarithm of the current sweep rate $\log v$.

In this paper, we derived the analytical expressions of the switching times $t_{\mathrm{sw}}$ for $b=1$ and $b=2$ due to the time dependent current $I(t)$, and calculated the dependence of the switching current $I\left(t_{\mathrm{sw}}\right)$ on the temperature $T$ and the sweep rate $v$. We found that $I\left(t_{\mathrm{sw}}\right)$ is approximately proportional to $T$ and $\log v$ for both $b=1$ and $b=2$ in the experimental ranges, and thus, the value of the exponent cannot be determined by the results of Ref. [12. We also found that a low temperature experiment will clarify the value of the exponent $b . I\left(t_{\mathrm{sw}}\right)$ is proportional to $T$ if $b=1$ while it is nonlinear to $T$ if $b=2$.

This paper is organized as follows. The analytical expressions of the switching probability $P$ and the switching time $t_{\mathrm{sw}}$ for $b=1$ and $b=2$ are derived in Sec. II and in Sec. III, respectively. The dependences of the switching current $I\left(t_{\mathrm{sw}}\right)$ on the temperature $T$ and the sweep rate $v$ are discussed in Sec. [V] Section $\mathrm{V}$ is the summary of this paper.

\section{SWITCHING PROBABILITY}

In this section, we derive the analytical expression of the magnetization switching probability due to the sweep current $(b=1$ and $b=2)$. We consider spin torque switching of the free layer magnetization in a ferromagnetic (fixed layer)/nonmagnetic/ferromagnetic (free layer) trilayer. Both ferromagnetic layers are assumed to have the perpendicular anisotropy along the $z$ axis. The 
magnetization $\mathbf{M}$ of the free layer is assumed to point to the positive $z$ direction at $t=0$. Throughout this paper, the applied field $H_{\text {appl }}$ is assumed to be zero for simplicity. From $t=0$, the current $I$ is applied to the ferromagnetic film perpendicular to plane and induces torque on the magnetization $\mathbf{M}$. The magnitude of the current $I$ is assumed to be smaller than the critical current $I_{\mathrm{c}}$. With the help of the thermal activation, the magnetization can change its direction to the negative $z$ direction. The switching probability of the magnetization, $P$, obeys the following equation [6]:

$$
\frac{\mathrm{d} P(t)}{\mathrm{d} t}=r(t)[1-P(t)]
$$

where $r(t)$ is the switching rate from the initial state $\left(\mathbf{M} \| \mathbf{e}_{z}\right)$ to the final state $\left(\mathbf{M} \|-\mathbf{e}_{z}\right)$. The explicit form of $r(t)$ is given by

$$
r(t)=f_{0} \exp \left[-\Delta_{0}\left(1-\frac{I(t)}{I_{\mathrm{c}}}\right)^{b}\right] .
$$

Here $b$ is the exponent of the current term $\left(1-I / I_{\mathrm{c}}\right)$. Although the attempt frequency depends on $I(t)$ in general, we approximate the value of $f_{0}$ as the attempt frequency at zero current. This approximation is applicable because the dependence of the switching rate $r(t)$ on the current $I(t)$ is mainly determined by the exponential term $\exp \left[-\Delta_{0}\left(1-I / I_{\mathrm{c}}\right)^{b}\right]$. The attempt frequency with zero current is given by $f_{0}=\alpha \gamma H_{\mathrm{K}} \sqrt{\Delta_{0} / \pi} /\left(1+\alpha^{2}\right)$, where $\alpha$ and $\gamma$ are the Gilbert damping constant and the gyromagnetic ratio, respectively [6].

Let us consider the thermally assisted magnetization switching due to time dependent current $I(t)$. As is in experiments [4, 12], we assume that the strength of $I(t)$ increases linearly with time, i.e., $I(t)=v t$, where $v$ is the sweep rate. By integrating Eq. (11) and using the initial condition $P(0)=0, P(t)$ is given by

$$
\begin{aligned}
& P(t)=1-\exp \left\{-\frac{f_{0} I_{\mathrm{c}}}{b v \Delta_{0}^{1 / b}}\right. {\left[\gamma\left(\frac{1}{b}, \Delta_{0}\right)\right.} \\
&\left.\left.-\gamma\left(\frac{1}{b}, \Delta_{0}\left(1-\frac{I}{I_{\mathrm{c}}}\right)^{b}\right)\right]\right\},
\end{aligned}
$$

where $\gamma(\beta, z)=\int_{0}^{z} \mathrm{~d} t t^{\beta-1} \mathrm{e}^{-t}$ is the incomplete $\Gamma$ function. For $b=1$ [7, 8] and $b=2$ [6, 9 11], $P(t)$ are, respectively, reduced to

$$
\begin{aligned}
& P_{1}(t)=1-\exp \left\{-\frac{f_{0} I_{\mathrm{c}}}{v \Delta_{0}}\right. {\left[\exp \left[-\Delta_{0}\left(1-\frac{v t}{I_{\mathrm{c}}}\right)\right]\right.} \\
&-\left.\left.\exp \left(-\Delta_{0}\right)\right]\right\}, \\
& P_{2}(t)=1-\exp \left\{-\frac{\sqrt{\pi} f_{0} I_{\mathrm{c}}}{2 v \sqrt{\Delta_{0}}}\left[\operatorname{Erf}\left(\sqrt{\Delta_{0}}\right)\right.\right. \\
&\left.\left.-\operatorname{Erf}\left(\sqrt{\Delta_{0}}\left(1-\frac{v t}{I_{\mathrm{c}}}\right)\right)\right]\right\},
\end{aligned}
$$

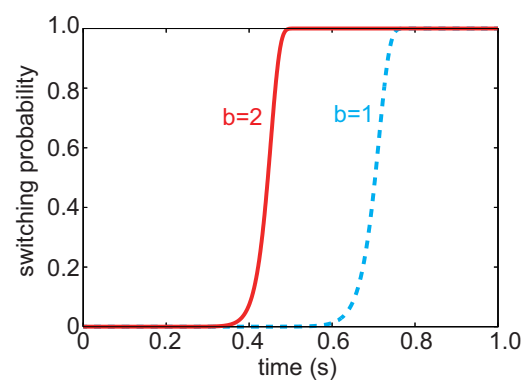

FIG. 1: The time evolutions of $P(t)$ for $b=1\left(P_{1}\right.$ in Eq. (4) $)$ and $b=2\left(P_{2}\right.$ in Eq. (5) ).

where $\operatorname{Erf}(z)=(2 / \sqrt{\pi}) \int_{0}^{z} \mathrm{~d} t \mathrm{e}^{-t^{2}}$ is the error function. Equations (4) and (5) are the main results in this section.

Figure 1] shows the time evolutions of $P_{1}$ and $P_{2}$ under the effect of the sweep current. The values of the parameters are taken to be $\alpha=0.007, \gamma=1.764 \times 10^{7}$ $\mathrm{Hz} / \mathrm{Oe}, H_{\mathrm{K}}=200 \mathrm{Oe}, M=995 \mathrm{emu} /$ c.c., $T=300$ $\mathrm{K}, d=2.0 \mathrm{~nm}$ and $S=\pi \times 80 \times 35 \mathrm{~nm}^{2}$, where $d$ and $S$ are the thickness and cross section area of the ferromagnetic layer, respectively, which are typical values for the Spin RAM cell consists of the transition ferromagnetic metals such as $\mathrm{CoFeB}[4,5]$. The thermal stability is defined by $\Delta_{0}=M H_{\mathrm{K}} V /\left(2 k_{\mathrm{B}} T\right)$, where $V=S d$ is the volume. The critical current $I_{\mathrm{c}}$ is given by $I_{\mathrm{c}}=[2 \alpha e M S d /(\hbar g)]\left(H_{\mathrm{K}}+2 \pi M\right)$ [13], where spin polarization $g$ is taken to be 0.5 . By using the above parameters, the values of $f_{0}, \Delta_{0}$, and $I_{\mathrm{c}} / S$ are given by 0.09 $\mathrm{GHz}, 42$, and $5.5 \times 10^{6} \mathrm{~A} / \mathrm{cm}^{2}$, respectively. The sweep rate is taken to be $v / S=5.0 \times 10^{6} \mathrm{~A} / \mathrm{cm}^{2} \mathrm{~s}(v=0.44$ $\mathrm{mA} / \mathrm{s}$ ), which is similar to the experimental values [12].

The switching probability $P$ suddenly changes its value from 0 to 1 at a certain time $t_{\mathrm{sw}}$, as shown in Fig. 1, in which $t_{\mathrm{sw}}$ satisfies $I\left(t_{\mathrm{sw}}\right)<I_{\mathrm{c}}$. In next section, we derive the analytical expression of the switching time $t_{\mathrm{sw}}$.

\section{SWITCHING TIME}

In this section, we derive the analytical expression of the switching time. At $t=t_{\mathrm{sw}}, \mathrm{d} P / \mathrm{d} t$ takes its maximum, as shown in Fig. 11 Thus, $P(t)$ satisfies $\mathrm{d}^{2} P / \mathrm{d} t^{2}=0$ at $t=t_{\mathrm{sw}}$. This condition can be rewritten as

$$
\frac{\mathrm{d} r}{\mathrm{~d} t}=r^{2}
$$

By using the explicit form of $r(t)$ (Eq. (2)), we find that

$$
\frac{b v \Delta_{0}}{I_{\mathrm{c}}}\left(1-\frac{I\left(t_{\mathrm{sw}}\right)}{I_{\mathrm{c}}}\right)^{b-1}=f_{0} \exp \left[-\Delta_{0}\left(1-\frac{I\left(t_{\mathrm{sw}}\right)}{I_{\mathrm{c}}}\right)^{b}\right] \text {. }
$$

In the case of $b=1$, Eq. (7) can be easily solved, and we find that

$$
\frac{I\left(t_{\mathrm{sw}}\right)}{I_{\mathrm{c}}}=1-\frac{1}{\Delta_{0}} \log \frac{f_{0} I_{\mathrm{c}}}{v \Delta_{0}} .
$$



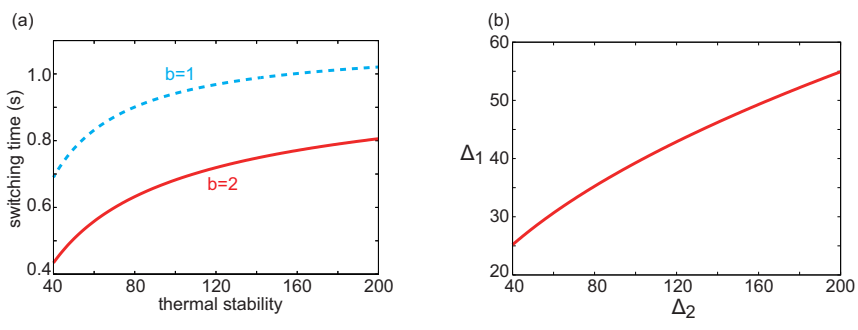

FIG. 2: (a) The dependences of the switching times for $b=1$ and $b=2$ (Eqs. (9) and (12)) on the thermal stability $\Delta_{0}$. (b) The relation between the thermal stabilities estimated by Eqs. (9) and (12).

By using $I=v t$, the switching time for $b=1$ is given by

$$
t_{\mathrm{sw} 1}=\frac{I_{\mathrm{c}}}{v}\left(1-\frac{1}{\Delta_{0}} \log \frac{f_{0} I_{\mathrm{c}}}{v \Delta_{0}}\right) \text {, }
$$

which is identical to that obtained by Li and Zhang [8] for $P=1-\mathrm{e}^{-1}$ (see Eq. (22) in Ref. [8]).

To obtain the explicit form of $t_{\mathrm{sw}}$ for general $b$, with the help of Eq. (8), let us consider the solution of $I\left(t_{\mathrm{sw}}\right) / I_{\mathrm{c}}$ whose form is given by

$$
\frac{I\left(t_{\mathrm{sw}}\right)}{I_{\mathrm{c}}}=1-\left(\frac{1}{\Delta_{0}} \log X\right)^{1 / b},
$$

where $X$ is determined by Eq. (77). By substituting Eq. (10) into Eq. (7), we find that $X$ satisfies $(\log X)^{1-1 / b}=1 /(C X)$, where $C=b v \Delta_{0}^{1 / b} /\left(f_{0} I_{\mathrm{c}}\right)$. In terms of $Y=\log X$, this equation can be expressed as $Y \mathrm{e}^{Y} Y^{-1 / b}=1 / C$, and its solution is given by $Y=$ $[(b-1) / b] \operatorname{plog}\left[b C^{-b /(b-1)} /(b-1)\right]$, where $\operatorname{plog}(z)$ is the product log (or Lambert $W$ function) which satisfies $\operatorname{plog}(z) \exp [\operatorname{plog}(z)]=z . I\left(t_{\mathrm{sw}}\right) / I_{\mathrm{c}}$ is thus given by

$$
\frac{I\left(t_{\mathrm{sw}}\right)}{I_{\mathrm{c}}}=1-\left\{\frac{b-1}{b \Delta_{0}} \operatorname{plog}\left[\frac{b}{b-1}\left(\frac{f_{0} I_{\mathrm{c}}}{b v \Delta_{0}^{1 / b}}\right)^{b /(b-1)}\right]\right\}^{1 / b} .
$$

In the case of $b=2$, the switching time $t_{\mathrm{sw} 2}$ is given by

$$
t_{\mathrm{sw} 2}=\frac{I_{\mathrm{c}}}{v}\left\{1-\sqrt{\frac{1}{2 \Delta_{0}} \operatorname{plog}\left[\left(\frac{f_{0} I_{\mathrm{c}}}{\sqrt{2} v \sqrt{\Delta_{0}}}\right)^{2}\right]}\right\} \text {. }
$$

Equations (9) and (12) are the main results in this section: these are the theoretical expressions of the switching times $t_{\mathrm{sw}}$, or equivalently, the switching current $I\left(t_{\mathrm{sw}}\right)=v t_{\mathrm{sw}}$. They are valid for the sweep rate $v$ which satisfies $t_{\mathrm{sw}}>0$. By using the parameter values listed in the previous section, $t_{\mathrm{sw} 1}$ and $t_{\mathrm{sw} 2}$ are estimated to be $0.71 \mathrm{~s}$ and $0.45 \mathrm{~s}$, respectively, which have good agreement with Fig. 1]

For a large $z, \operatorname{plog}(z)$ can be approximated to $\log (z)$. In this limit, Eq. (11) is reduced to

$$
\frac{I\left(t_{\mathrm{sw}}\right)}{I_{\mathrm{c}}} \simeq 1-\left\{\frac{1}{\Delta_{0}} \log \left[\left(\frac{b}{b-1}\right)^{1-1 / b} \frac{f_{0} I_{\mathrm{c}}}{b v \Delta_{0}^{1 / b}}\right]\right\}^{1 / b} .
$$

Neglecting a small correlation term $[b /(b-1)]^{1-1 / b}$, Eq. (13) is identical to the result of Garg [14] on the mean switching current $\langle I\rangle / I_{\mathrm{c}}=1-$ $\left\{\left(1 / \Delta_{0}\right) \log \left[f_{0} I_{\mathrm{c}} /\left(b v \Delta_{0}^{1 / b}\right)\right]\right\}^{1 / b}$ which was used in the analysis of the experimental result of Ref. [12].

Experimentalists [4, 12] repeat the measurement of the switching due to sweep current or magnetic field and measure the switching time of each trial. By fitting the average of the switching time with Eqs. (9) or (12), one can estimate the thermal stability $\Delta_{0}$. Figure2 (a) shows the dependence of the switching times, Eqs. (9) and (12), on the thermal stability $\Delta_{0}$, in which $\Delta_{0}$ is regarded as the independent variable from $I_{\mathrm{c}}$, as is in experiments [4, 5]. For example, when the switching time is about 0.8 s, the thermal stability estimated by Eq. (9) is about 50 while that estimated by Eq. (12) is about 200. Figure 2 (b) shows the relation between the thermal stabilities estimated by Eqs. (9) $\left(\Delta_{1}\right)$ and (12) $\left(\Delta_{2}\right)$ which can be obtained by putting $t_{\mathrm{sw} 1}\left(\Delta_{0}=\Delta_{1}\right)=t_{\mathrm{sw} 2}\left(\Delta_{0}=\Delta_{2}\right)$ and is given by

$$
\begin{aligned}
\Delta_{1} & =\sqrt{2 \Delta_{2}} \operatorname{plog}\left\{\frac{f_{0} I_{\mathrm{c}}}{\sqrt{2} v \sqrt{\Delta_{2}}} \sqrt{\operatorname{plog}\left[\left(\frac{f_{0} I_{\mathrm{c}}}{\sqrt{2} v \sqrt{\Delta_{2}}}\right)^{2}\right]}\right\} \\
& / \sqrt{\operatorname{plog}\left[\left(\frac{f_{0} I_{\mathrm{c}}}{\sqrt{2} v \sqrt{\Delta_{2}}}\right)^{2}\right]} \\
& \simeq \sqrt{\Delta_{2}} \sqrt{\log \frac{f_{0} I_{\mathrm{c}}}{\sqrt{2} v \sqrt{\Delta_{2}}}} .
\end{aligned}
$$

As shown in Fig. 2 (b), the difference of the exponent $b$ leads to a significant underestimation of the thermal stability.

\section{DEPENDENCE OF SWITCHING TIME ON TEMPERATURE AND SWEEP RATE}

Myers et al. experimentally studied the dependences of the switching current, $I\left(t_{\mathrm{sw}}\right)$, on the temperature $T$ and the sweep rate $v$ (see Fig. 1 (d) in Ref. [12]), and showed that $I\left(t_{\mathrm{sw}}\right)$ is approximately proportional to both $T$ and $\log v$. Reference 8] argued that these results support the theory with $b=1$ [7, 8] because such dependences are expected from Eq. (8). In this section, we study the dependences of $I\left(t_{\mathrm{sw}}\right)$ for $b=1$ and $b=2$ on $T$ and $v$ in and out of the experimental ranges.

The dependence of $I\left(t_{\mathrm{sw}}\right)$ on the temperature can be taken into account through the thermal stability $\Delta_{0}=$ $M H_{\mathrm{K}} V /\left(2 k_{\mathrm{B}} T\right)$. For simplicity, we neglect the dependence of the attempt frequency $f_{0} \propto \sqrt{\Delta_{0}}$ on the temperature $T$, as done in Ref. [8]. The solid (red) and dotted (blue) lines in Fig. 3 show the dependences of $I\left(t_{\mathrm{sw}}\right) / I_{\mathrm{c}}$ on $T$ for $b=1$ and $b=2$, respectively. The temperature range $(100-300 \mathrm{~K})$ is sufficient to consider the $I\left(t_{\mathrm{sw}}\right)$ in the experimental range (180-220 K in Ref. [12]). Even in 


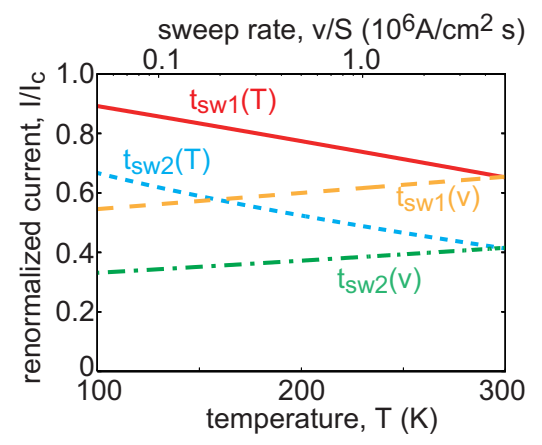

FIG. 3: The solid (red) and dotted (blue) lines are the dependences of $I\left(t_{\mathrm{sw}}\right) / I_{\mathrm{c}}$ on the temperature $T$ for $b=1$ and $b=2$, respectively. The dashed (orange) and dashed-dotted lines are the dependences of $I\left(t_{\mathrm{sw}}\right) / I_{\mathrm{c}}$ on the sweep rate $v$ for $b=1$ and $b=2$, respectively.
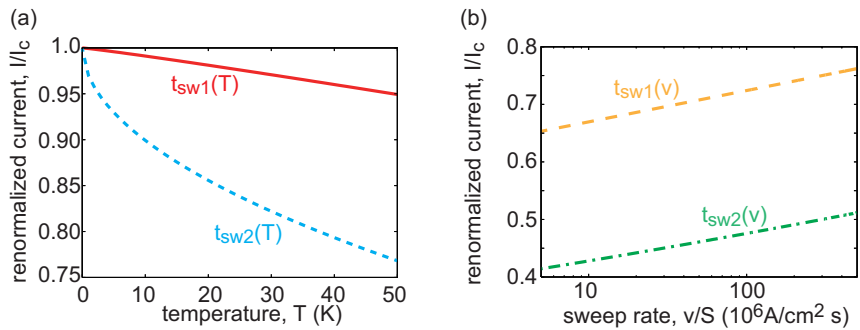

FIG. 4: The dependences of $I\left(t_{\mathrm{sw}}\right) / I_{\mathrm{c}}$ on (a) temperature $T$ and (b) the sweep rate $v$ out of the experimental range of Ref. [12].

such wide range of the temperature, both $I\left(t_{\mathrm{sw}}\right) / I_{\mathrm{c}}$ for $b=1$ and $b=2$ are approximately proportional to the temperature $T$. The dependences of $I\left(t_{\mathrm{sw}}\right) / I_{\mathrm{c}}$ on $v$ for $b=1$ and $b=2$ are also plotted by the dashed (orange) and dashed-dotted (green) lines in Fig. 3, respectively. The temperature is fixed to $T=300 \mathrm{~K}$. The range of $v / S$, from 0.05 to $5.0 \times 10^{6} \mathrm{~A} / \mathrm{cm}^{2} \mathrm{~s}$ (from 0.0044 to 0.44 $\mathrm{mA} / \mathrm{s}$ ), is very similar to that studied in experiments (from 0.01 to $1.0 \mathrm{~mA} / \mathrm{s}$ ). As shown in Fig. 3, both $I\left(t_{\mathrm{sw}}\right) / I_{\mathrm{c}}$ for $b=1$ and $b=2$ are approximately proportional to $\log v$. Summarizing the above results, the value of the exponent $b(b=1$ or $b=2)$ cannot be determined by the dependences of $I\left(t_{\mathrm{sw}}\right) / I_{\mathrm{c}}$ on the temperature $T$ and the sweep rate $v$ in the experimental range.

We also study the dependences of $I\left(t_{\mathrm{sw}}\right) / I_{\mathrm{c}}$ on $T$ and $\log v$ out of the experimental ranges of Ref. [12]. Figure 4 (a) shows the dependence of $I\left(t_{\mathrm{sw}}\right) / I_{\mathrm{c}}$ on $T$ in the low temperature region, $0<T \leq 50 \mathrm{~K}$. In this range, we can see that $I\left(t_{\mathrm{sw}}\right) / I_{\mathrm{c}}$ is approximately proportional to $T$ for $b=1$ while $I\left(t_{\mathrm{sw}}\right) / I_{\mathrm{c}}$ for $b=2$ is not. Thus, to determine the exponent $b$, experiments should be performed in such very low temperature region. The dependences of $I\left(t_{\mathrm{sw}}\right) / I_{\mathrm{c}}$ on $\log v$ for both $b=1$ and $b=2$ are approximately proportional to $\log v$ even in the large sweep rate region up to $v / S=500 \times 10^{6} \mathrm{~A} / \mathrm{cm}^{2} \mathrm{~s}$, as shown in Fig. 4 (b), and thus, it is difficult to determine $b$ from these dependences.

\section{SUMMARY}

In summary, we studied the dependence of the spin torque switching probability on the sweep current. The analytical expressions of the switching time for both $b=1$ and $b=2$ are derived, where $b$ is the exponent of the current term in the switching rate. We showed that $I\left(t_{\mathrm{sw}}\right) / I_{\mathrm{c}}$ for $b=1$ and $b=2$ are approximately proportional to the temperature $T$ and the logarithm of the sweep rate $v$ in the experimental ranges of $T$ and $v$, and thus, the value of the exponent $b$ cannot be determined by the dependences of $I\left(t_{\mathrm{sw}}\right) / I_{\mathrm{c}}$ on $T$ and $\log v$. We also showed that a low temperature experiment is required to determine the exponent $b . I\left(t_{\mathrm{sw}}\right)$ is proportional to $T$ if $b=1$ while it is nonlinear to $T$ if $b=2$.

\section{Acknowledgments}

The authors would like to acknowledge S. Yuasa, H. Kubota, S. Yakata, D. Bang, T. Saruya, T. Yorozu, K. Seki, M. Marthaler, and H. Sukegawa for the valuable discussions they had with. This work was supported by JSPS and NEDO.
[1] J. C. Slonczewski, J. Magn. Magn. Mater. 159, L1 (1996).

[2] L. Berger, Phys. Rev. B 54, 9353 (1996).

[3] J. Hayakawa, S. Ikeda, K. Miura, M. Yamanouchi, Y. M. Lee, R. Sasaki, M. Ichimura, K. Ito, T. Kawahara, R. Takemura, et al., IEEE. Trans. Magn. 44, 1962 (2008).

[4] S. Yakata, H. Kubota, T. Seki, K. Yakushiji, A. Fukushima, S. Yuasa, and K. Ando, IEEE Trans. Magn. 46, 2232 (2010).

[5] S. Yakata, H. Kubota, T. Sugano, T. Seki, K. Yakushiji, A. Fukushima, S. Yuasa, and K. Ando, Appl. Phys. Lett. 95, 242504 (2009).

[6] W. F. B. Jr, Phys. Rev. 130, 1677 (1963).

[7] R. H. Koch, J. A. Katine, and J. Z. Sun, Phys. Rev. Lett. 92, 088302 (2004).
[8] Z. Li and S. Zhang, Phys. Rev. B 69, 134416 (2004).

[9] Y. Suzki, A. A. Tulapurkar, and C. Chappert, Nanomagnetism and Spintronics (Elsevier, 2009), Chapter 3.

[10] T. Taniguchi and H. Imamura, Phys. Rev. B 83, 054432 (2011).

[11] Private communication with C. Mewes. Their results were recently presented at $55^{\text {th }}$ Annual Conference on Magnetism and Magnetic Materials by W. Butler (presentation number is HC-09).

[12] E. B. Myers, F. J. Albert, J. C. Sankey, E. Bonet, R. A. Buhrman, and D. C. Ralph, Phys. Rev. Lett. 89, 196801 (2002).

[13] J. Z. Sun, Phys. Rev. B 62, 570 (2000).

[14] A. Garg, Phys. Rev. B 51, 15592 (1995). 